\title{
RESEARCH
}

\section{Topino: A graphical tool for quantitative assessment of molecular stream separations}

\author{
Sven Kochmann, Nikita A Ivanov and Sergey N Krylov*
}

\begin{abstract}
In molecular-stream separation (MSS), a stream of a multi-component mixture is separated into multiple streams of individual components inside a thin rectangular chamber. Despite great potential and many years of work on MSS, its analysis was underdeveloped until recently. To fill in this gap, we introduced a novel and convenient way to assess MSS by convoluting the separation zone into a simple 2D plot called angulagram. We implemented and publicly shared Python programs for the generation of angulagrams. However, we realized that Python programs create two hurdles (setting up a resembling Python environment and using a command line interface) for other researchers to try out and adopt this new approach. To make our approach more accessible to the MSS community, we introduce and present here the open-source software Topino for quantitative assessment of MSS. Topino is a user-friendly, accessible program with a graphical user interface that allows the user to assess MSS data in a fast and straightforward way (less than $2 \mathrm{~min}$ ). The software is available at https://github.com/Schallaven/topino.
\end{abstract}

Keywords: Analytical methods; Angulagrams; Molecular stream separations; continuous flow electrophoresis; Continuous annular chromatography; Free flow electrophoresis; Cheminformatics; Open source software; Graphical user interface (GUI); Model-View-Controller (MVC)

\section{Introduction}

In molecular-stream separation (MSS) a stream of a multi-component mixture is separated into multiple streams of individual components inside a rectangular chamber (Fig. 1). MSS can be achieved by chromatographic or electrophoretic means; the methods are called i.e. continuous annular chromatography and continuous flow electrophoresis (also called free flow electrophoresis), respectively [1-3]. MSS has great potential for applications that require continuous and seamless downstream separation such as continuous flow synthesis [4-7]. Despite great potential and many years of work on MSS methods themselves, the analysis of streams, i.e. the central element of MSS, was underdeveloped until recently [8-17]. We developed a set of tools for the quantitative characterization of streams in MSS to fill in this gap $[18,19]$. We introduced a novel and convenient way to assess MSS by convoluting the separation zone into a simple 2D plot called angulagram (Fig. 1). Convolution is done by exchanging the

\footnotetext{
${ }^{*}$ Correspondence: skrylov@yorku.ca

Department of Chemistry and Centre for Research on Biomolecular Interactions, York University, 4700 Keele Street, M3J 1P3 Toronto, ON, Canada

Full list of author information is available at the end of the article
}

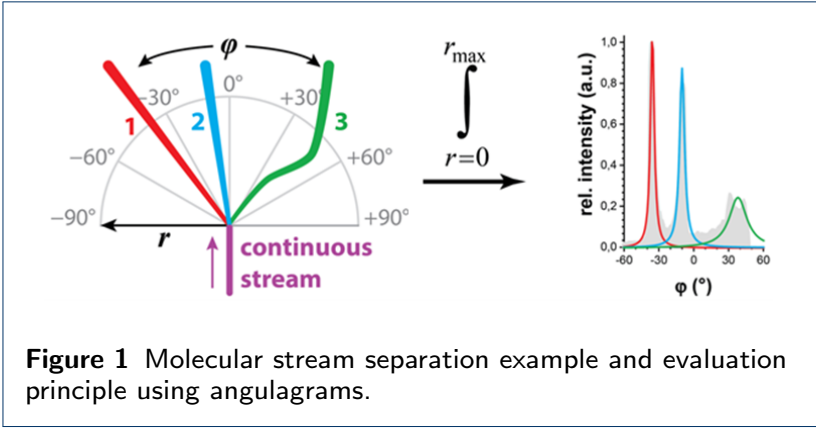

common Cartesian coordinate system for a polar coordinate system with origin at the inlet and subsequent integration over the radius. In order to allow other researchers to try out and eventually adopt this new approach we created and publicly shared our Python programs, which have already been adopted by at least one other group [20-24].

However, we realized that Python programs create two hurdles for other researchers to try out and adopt this new approach. First, they require a running and configured Python environment that resembles the development environment (same Python packages, etc.). Creating, installing, and maintaining a Python envi- 
ronment can be a cumbersome task on some platforms such as Windows, in particular, for inexperienced Python users. Second, all of our developed programs require to input precise parameters by a command line interface (CLI). CLI programs are powerful tools for advanced power users, but discourage new users due to the unfamiliar way of interaction and lack of visualization of processes.

In order to tackle these two issues and lower the overall hurdle towards using and adopting angulagrams for assessing MSS, we introduce Topino here. The goal of the development of Topino was to create a userfriendly, accessible program that allows the user to assess MSS raw data, i.e. images, in a fast and straightforward way. To achieve this goal, we used a modern version of $\mathrm{C}++$ in combination with the $\mathrm{Qt}$ framework library and Eigen 3 math library [25, 26]. The result is an easy-to-distribute program with a modern and clear dark-themed graphical user interface (GUI) that runs on all current versions of major operating systems (Windows, Linux, and Mac).

\section{Implementation}

\section{Design criteria for Topino}

Design criteria were selected by examining all necessary tasks involved in quantitative assessment of MSS. In general, an experiment has to be performed and at least one image (= raw data) of this experiment has to be recorded. Technically and in most cases, an image is a multicolor picture file (e.g. jpeg or png with 3 channels of colors) acquired by a digital camera and its respective software. Each image has to be assessed individually by conducting the following steps [19]. First, the parameters of the polar coordinate system (origin, direction of the $0^{\circ}$ angle, boundaries of radius and angle) have to be chosen dependent on the region-ofinterest (ROI) on the image. The ROI is defined by the position of the inlet, the direction and position of the streams, and the boundary of the separation zone. Second, the image has to be converted to a grayscale image (single color channel). In this grayscale image, the color values represent the signal intensities of the molecules in the streams; low color values (i.e. closer to black) represent low signal intensities (background) and high color values (i.e. closer to white) represent high signal intensities, respectively. Third, the ROI has to be extracted as a polar coordinate-based image and, subsequently, be convoluted by integrating over the radius axis to gain an angulagram (i.e. angle vs signal intensity). Last, the peaks in the angulagram have to be fitted by a Lorentzian function to gain the stream parameters (stream deflection, stream width, stream linearity, and stream resolution).
In order to support the user in these steps to full extent, the development of Topino followed some specific design criteria. First, Topino should provide an accessible and clear user interface, which allows to manipulate items such as the coordinate system by simple mouse interaction. Second, Topino should establish and facilitate a natural and logical workflow that follows the image assessment steps above. Third, data should be stored in an human-readable format as well as be easy to transfer to other programs. Last, Topino should be easy to deploy and run without further configuration beyond a simple installation process. The following paragraphs describe the implementation of Topino regarding the assessment steps and design criteria.

\section{General implementation of Topino}

Topino is a desktop application and uses the Qt framework with a model-view-controller (MVC) pattern to setup its basic structure. The model is implemented in the classes TopinoDocument and TopinoData handling the overall functionality of file handling and the actual data, respectively. Topino uses an XMLbased structure with Base64 encoded images for its files. These files are human-readable and their content can be easily transfered to other programs. There are two views implemented in Topino, one for working with the image (setting up the coordinate system, etc.) and one for working with the angulagram (fitting peaks, etc.). These two views are implemented in the classes ImageAnalysis View and AngulagramView (both derived from an abstract class TopinoAbstractView), respectively. Finally, the controller is mainly implemented in the Main Window class encapsulating the Topino window and most of the user interface itself, which uses a single-document-interface (SDI) to present data and information to the user in a clear and accessible way (Fig. 2).

\section{Creating the polar coordinate system}

A central interface element in Topino is the inlet tool that allows users to define and manipulate the polar coordinate system and its parameters (Fig. 3). The polar coordinate system is represented by a small inner circle (the actual inlet) and a larger outer circle. Both circles have the exact same center position that defines the origin of the inlet and polar coordinate system. The visible part of the outer circle defines the ROI by its $0^{\circ}$ angle direction, minimum/maximum angle, and minimum/maximum radius. All these elements can be manipulated by simply clicking in or on them and dragging with the mouse. For instance, the position of the origin can be changed by clicking into and dragging the area of the smaller circle. Likewise, the minimum 


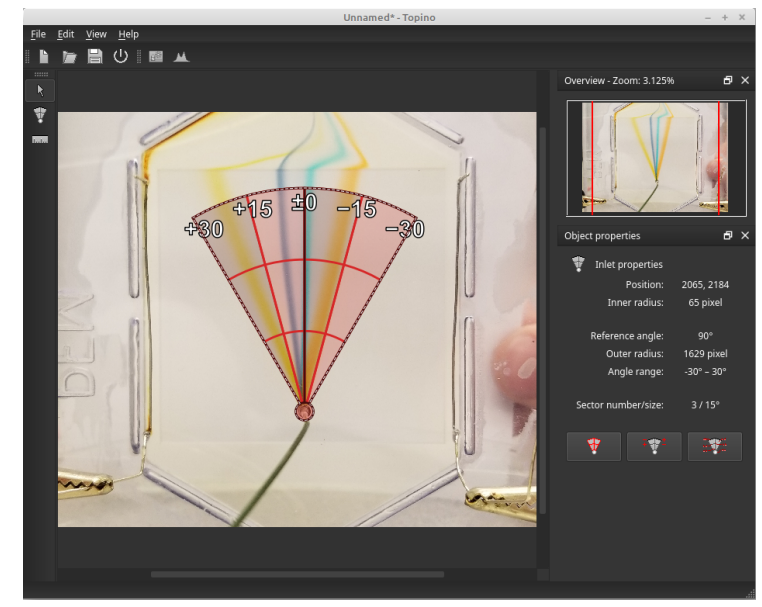

Figure 2 Main window of Topino showing an overview of its GUI.

radius or the $0^{\circ}$ angle direction can be changed by clicking and dragging the border of the inner circle or the $0^{\circ}$ angle line, respectively.

Only the ROI area is used to generate the angulagram, i.e. all pixels inside the smaller circle as well as all pixels outside of the whole polar coordinate system representation are ignored. This implementation of ROI focusing allows us to remove unnecessary background such as the non-signal pixels of the physical inlet as seen on the example picture in (Fig. 3) and avoids potential data-changing preprocessing steps such as cutting or rotating the image.

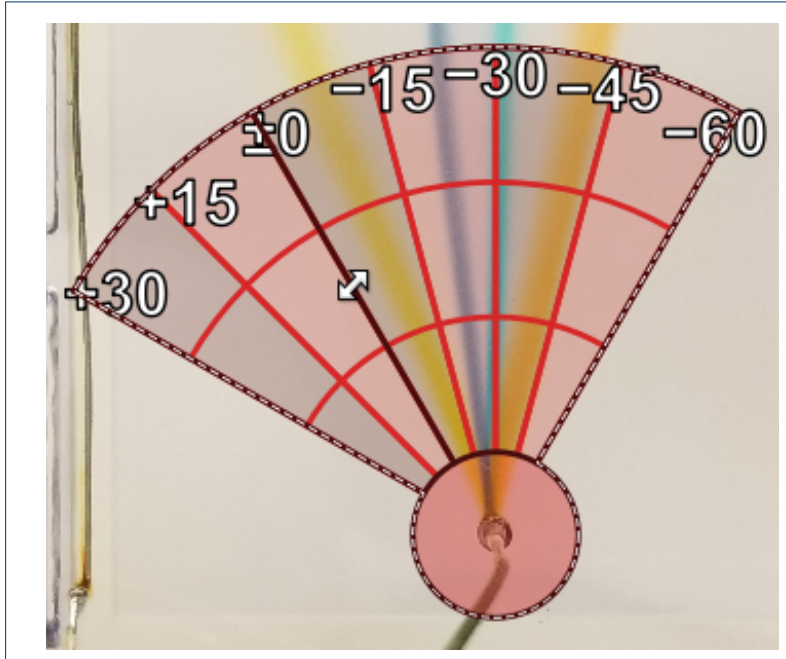

Figure 3 Inlet tool of Topino allows to change its parameters by simply clicking and dragging.

\section{Conversion to a grayscale image}

A pixel color in an image is a combination of three colors red, green, and blue (RGB). Topino supports standard 24 bit images, i.e. 8 bit per channel, and will implicitly convert every image imported into 24 bit. The conversion of a color image into a grayscale image is converting these RGB values (24 bit image) into a single grayscale value ( 8 bit image). This conversion process is called decolorization or desaturation [27]. Typically, a linear combination of RGB values is used to generate a grayscale value, e.g. by calculating and using the luminance of a pixel by

$$
\begin{array}{r}
L(x, y)=0.21 \times R(x, y)+0.72 \times G(x, y)+ \\
0.07 \times B(x, y)
\end{array}
$$

where $x$ and $y$ are the pixel coordinates in Cartesian coordinates. $R, G$, and $B$ are the red, green, and blue values at $(x, y)$ resulting in the luminance value $L$, which is used as gray value at $(x, y)$.

There are several conversion methods to choose from and the best applicable method depends strongly on the image. Therefore, we implemented an accessible WYSIWYG (what-you-see-is-what-you-get) dialog in which the user can select from different conversion methods and see the effect on the ROI immediately (Fig. 4). Moreover, the gray values (= signal intensities) can be inverted to ensure that low signal intensity equals low gray value and high signal intensity equals high gray value. Finally, the gray value levels can be filtered by adjusting the boundaries of the histogram to remove unwanted noise and background. All gray values outside of this filter will be set to zero.

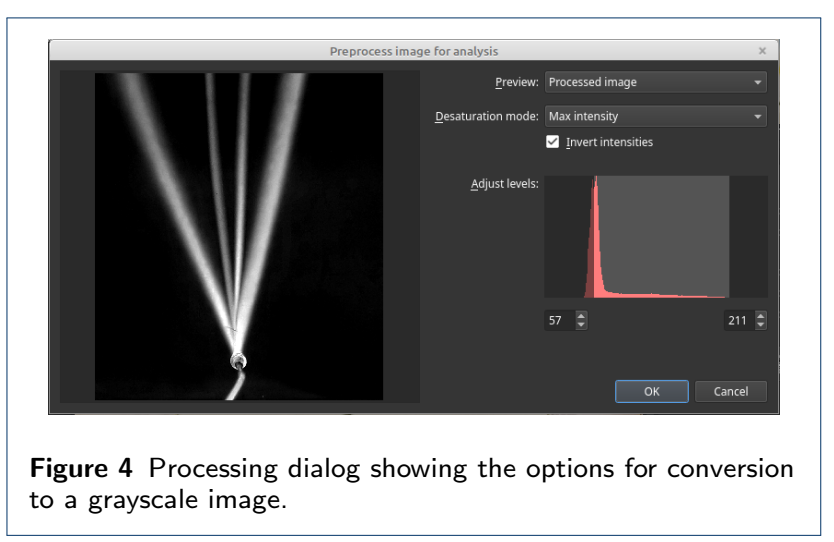

Extraction of ROI and generation of angulagram

ROI has to be extracted as a polar coordinate-based image (short: polar image) from the grayscale image and, subsequently, be convoluted by integrating over 
the radius axis to gain an angulagram. Internally, Topino creates a polar image automatically whenever necessary, e.g. when the user changes the grayscale conversion method (see above). The calculation of the polar image is done by iteration over all radii $r$ (1 pixel steps) and angle $\phi$ values $\left(0.1^{\circ}\right.$ steps $)$ in their respective boundaries (as defined by the ROI) and assigning the gray value intensity from a point $(x, y)$ of the grayscale image. $x$ and $y$ are calculated as

$$
\begin{aligned}
& x=x_{0}+\operatorname{round}(r \times \cos (\phi)) \\
& y=y_{0}-\operatorname{round}(r \times \sin (\phi))
\end{aligned}
$$

where $x_{0}$ and $y_{0}$ are the coordinates of the origin. $\operatorname{round}(\ldots)$ is a function that rounds a real value to the nearest integer since pixel coordinates are integers. The generated polar image can be viewed by pressing the respective button in the angulagram view.

Subseqently, this polar image is convoluted by integration over the radius to generate the angulagram. The intensity $I$ at angle $\phi$ is calculated by

$$
I(\phi)=\int_{r_{\min }}^{r_{\max }} I(\phi, r) d r \approx \sum_{r=r_{\min }}^{r_{\max }} I(\phi, r)
$$

where $r_{\min }$ and $r_{\max }$ are the radii boundaries of the ROI and, thus, of the polar image. $I(\phi, r)$ is the intensity in the polar image at the respective angle and radius. Internally discrete values are used for $\phi$ and $r$ due to the integer representation of pixel coordinates. Therefore, the integration is basically the same as the summation over all $r$ for a given $\phi$. The plotted angulagram $I(\phi)$ is presented to the user in the angulagram view (Fig. 5). For convenience and clarity, the view uses relative intensity to display the angulagram. Exported data will reveal the absolute intensity values. The calculation of the angulagram is (re)done whenever necessary or the user switches to the angulagram view.

\section{Extracting stream parameters from an angulagram}

Each peak in the angulagram represents a streams. In our previous study we found that a prefect linear streams resembles a Lorentzian function in the angulagram. Such a Lorentzian function $F$ can be defined as

$$
F(\phi)=h \frac{w^{2}}{w^{2}+\left(\phi_{0}-\phi\right)^{2}}+F_{0}
$$

where $h, w$, and $\phi_{0}$ are the height, width, and position of the peak, respectively. $F_{0}$ is the shift along the intensity axis of $F$. Fitting this function to a peak in the angulagram gains the respective peak parameters.

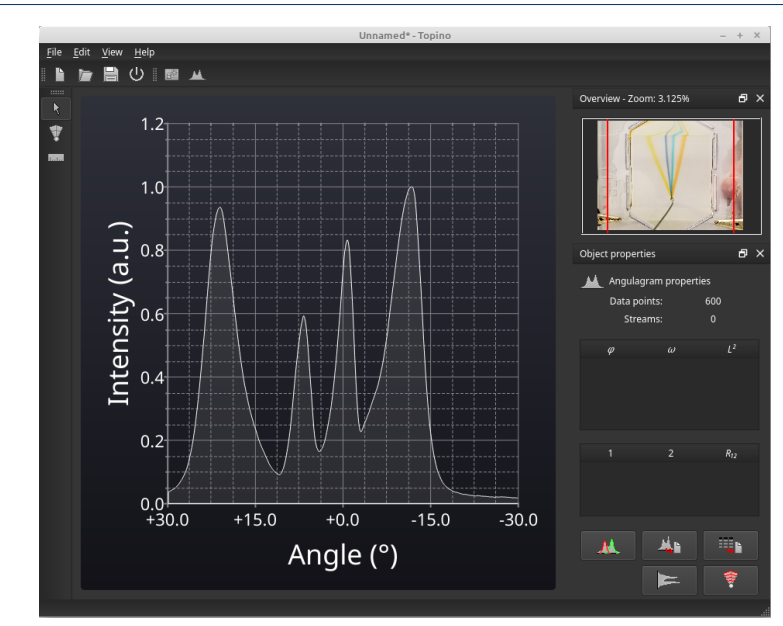

Figure 5 Angulagram view of Topino.

Subsequently, stream parameters can be calculated, i.e. stream deflection, stream width, stream linearity, and stream resolution for any pair of streams. Stream deflection and stream width are simply $\phi_{0}$ and $w$ of the peak, respectively. The stream linearity $L^{2}$ is characterized by the closeness of the fit $F(\phi)$ to the actual data $P(\phi)$ expressed by R-squared (coefficient of determination) determinded by least-squares fitting (to not confuse $R^{2}$ with resolution we use $L^{2}$ for linearity here):

$$
L^{2}=\left(\frac{\sum\left(p_{d}-\overline{p_{d}}\right)\left(f_{d}-\overline{f_{d}}\right)}{\sqrt{\sum\left(p_{d}-\overline{p_{d}}\right)^{2}\left(f_{d}-\overline{f_{d}}\right)^{2}}}\right)^{2}
$$

where $f_{d}$ and $p_{d}$ are discrete data points of $F$ and $P$, respectively, and $\overline{f_{d}}$ and $\overline{p_{d}}$ are the respective averages of these data points. Only data points in a certain boundary that belong to the respective steam are used for determinig $L^{2}$. Finally, stream resolution of two streams is defined as

$$
R_{12}=\frac{2 \times\left|\phi_{2}-\phi_{1}\right|}{w_{1}+w_{2}}
$$

where the subscripts designate the respective peak numbers.

In Topino fitting is done using the LevenbergMarquardt algorithm implemented in the Eigen 3 math library [26]. Maxima and minima in the angulagram are determined and used as boundaries for the peak fits. The fitting process is visualized to the user in a separated dialog window (Fig. 6). This dialog window allows changing fitting parameters like the smoothing degree or threshold value. Changing any value will update the current fit on the left side of the dialog window giving immediate feedback to the user. 


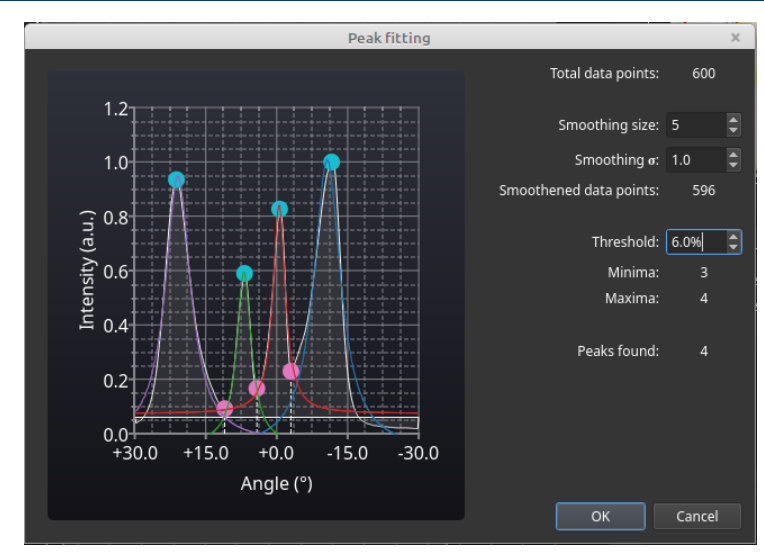

Figure 6 Dialog showing the angulagram fitting options with a live view.

\section{Exporting data}

MSS researchers and developers assess images and data to make conclusions about their work on or with MSS. Thus, Topino is highly likely not the last step in the data processing workflow since extracted stream parameters, angulagrams, or images need to be further processed or presented (e.g. in a talk or the like). Therefore, it is crucial that after evaluation in Topino, the user is able to export data in various formats in a straightforward way.

We implemented two general ways of exporting. First, classical export functions by menu or the respective object property pages allow the user to save the current viewed image, graph, or underlying data as raster or vector image as well as textual information that can be imported into other applications such as graphic or spreadsheet programs. Second, the user can simply press $\mathrm{Ctrl}+\mathrm{C}$ in either in image or angulagram view to copy data to the clipboard. The data will be either represented as an image or text depending on the application the user pastes the data into; graphic programs will receive the image and text editors the underlying data values. Some applications allow to choose which format to import by providing a 'Paste special...' function or the like. This second way of exporting provides users of Topino a seamless data processing workflow without intermediate step of file export and subseqent import.

\section{General workflow in Topino}

All the previous implementations were optimized and tailored towards a short and straightforward general workflow as described as follows. After starting Topino, the user is presented with an empty workspace. First, an image has to be imported by File $\rightarrow$ Import Image. Second, a main inlet has to be created by the inlet tool that defines the source and parameters of the polar coordinate system. Third, the desaturation mode and color level boundaries for the image need to be choosen to ensure the correct generation of an angulagram. Fourth, the angulagram is generated and evaluated by fitting the peaks to extract the respective stream parameters. Last, the resulting stream parameters can be exported or simply copied to other applications for presentation or further processing. The whole process takes less than $2 \mathrm{~min}$ for an user that has gone through this workflow once or twice in Topino.

\section{Additional features and functions}

There are more features and functions in Topino than the previously presented ones. For instance, there is a ruler tool beside the inlet tool allowing to define lines on an image. These lines can be snapped to density points on the image and, thus, used to precisely align the parameters of the polar coordinate system to features on the image. As another example, a radialgram can be generated and displayed. A radialgram integrates the polar image over the angle instead of the radius, which can be used to follow the progression and dispersion of streams through the ROI. A typical radialgram is shaped as a half-peak that follows progressive loss of signal intensity due to molecular diffusion of streams. Any deviations from this expected shape can point to debris found on the MSS device (sudden narrow drops), non-uniformity in lightning or exposure (overall curve shape change), or errors in preprocessing of the image (random peaks or drops). All these advanced functions are not needed for every day assessment of MSS but are meant as power features for advanced users that need an extra bit more control and precision for difficult-to-assess border cases.

\section{Results and discussion}

In this section, we compare Topino with our previously published Python programs [21] regarding its setup effort, usability, and evaluation results.

\section{Setup}

As mentioned in the Introduction section, our previous Python programs require a running and configured Python environment. In most Linux distributions there is already a Python environment available and setting up requires only to ensure that the requires Python packages are all available and installed in the right versions. In Windows, however, there is no Python environment preinstalled and the user has to install a new one. Apart from setting up the environment, our Python programs just have to be downloaded and put in a directory that is included in the search path (PATH environment variable of the operating system), so they can be run from anywhere. For 
any advanced user, these steps are not a problem. For an inexperienced user, these steps read cumbersome and are in fact a major hurdle that will lead to skip trying out a new program/approach.

On the other side, Topino can be installed by either using the setup program (Windows), the Debian package (Linux), or extracting the respective archive to any desired place (all operating systems). All required Qt libraries are installed by the setup program or package manager allowing the user to run Topino right away by simply double clicking on the executable. Installing programs in this way is common to even the most inexperienced user providing easy access to Topino and its MSS assessment functionality.

In conclusion, the setup approach is a fast and accessible experience leading more likely to a new user installing and trying out Topino. The Python programs are only accessible if a Python environment is already setup from an experienced user.

\section{Usability}

Our Python programs use a CLI. A typical command line to evaluate an input image input.jpg with our Python program angureflexin.py to create an angulagram looks like

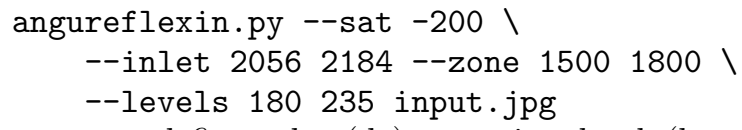

where --sat defines the (de)saturation level (here: -200), --inlet and --zone the inlet position (here: $x=2056, y=2184$ ) and ROI (here: a rectangular zone with 1500 pixels in width and 1800 pixels in height with the inlet at the bottom middle, i.e. from $(1306,384)$ to $(2806,2184))$ in Cartesian coordinates, and --levels the gray values (here: all gray values between 180 and 235) used for evaluation. The respective values have to be determined manually by a graphics program such as Adobe Photoshop or the like. These Python programs are extremely versatile and flexible. For instance, input and output can be redirected by piping and programs can be included easily in complex batch processes making it possible to evaluate thousands of images as demonstrated by us before [22]. However, a new user is easily overwhelmed and discouraged by the parameters needed to evaluated an image as well as the manual process to determine the input parameters rendering this CLI-based implementation less accessible.

In contrast and as described in the Implementation section, Topino uses a clear and modern GUI that resembles a typical graphics application. It guides the user by a logical workflow (see above), meaningful icons, descriptive text, and visualization of process results along the workflow. Moreover, Topino is developed to be "complete", i.e. the user can get all necessary information, parameters, and evaluation data inside of Topino and no other program is required. While Topino increases the accessibility of MSS evaluation, it is less flexible and versatile. For instance, automation and batch evaluation is not straightforward to implement and, therefore, not available currently.

In conclusion, the Python programs are powerful, versatile, and flexible tools for MSS evaluation but lack accessibility, guidance, and visualization. Topino is not as versatile and flexible but provides high accessiblity, guidance, and visualization.

\section{Evaluation results}

Unsurprisingly, Topino and our Python programs lead to comparable results when evaluating the same image due to the same underlying approach. For instance, a quick re-evaluation of the input image of Figure $2 \mathrm{~A}$ in Ref. 22 with Topino lead to stream deflections $-10.8^{\circ},-0.8^{\circ},+6.6^{\circ}$, and $+20.5^{\circ}$ (Python: $-12.5^{\circ}$, $-1.6^{\circ},+6.4^{\circ}$, and $\left.+21.2^{\circ}\right)$, stream widths $8.3^{\circ}, 3.1^{\circ}$, $3.8^{\circ}$, and $7.3^{\circ}$ (Python: $8.0^{\circ}, 2.5^{\circ}, 3.2^{\circ}$, and $6.2^{\circ}$ ) as well as stream linearities $L^{2} \geq 0.95$ (Python: $L^{2} \geq 0.95$ ). The differences are mainly due to minor improvements and additional features in Topino such as being able to select a lower boundary for the radius of the ROI and more grayscale conversion methods that are better applicable to this input image.

\section{Contributions and further development}

The development of Topino is not finished. While the current release version 1.0 of Topino includes everything needed to assess and evaluate MSS images with angulagrams as well as some more advanced functions, we want to add more functionality over the next months. For instance, we want to include a batch function to semi-automatically process a set of files and the ability to disect complex peaks as described in our previous publication [19].

For this development, we want to encourage and invite everyone who is interested to contribute ideas, code, and bug reports to our public software repository at https://github.com/Schallaven/topino.

\section{Conclusions}

Here, we introduced and presented Topino, an userfriendly, accessible program. We used a modern version of $\mathrm{C}++$ in combination with the Qt framework library and Eigen 3 math library to develop Topino resulting in an easy-to-distribute program with a modern and clear dark-themed graphical user interface (GUI). Topino allows to assess MSS data in a fast and straightforward (less than $2 \mathrm{~min}$ ) way using angulagrams. It does not possess the significant hurdles from our previous published Python programs. Topino's setup approach is a fast and accessible experience leading more likely 
to a new user installing and trying out Topino. While Topino is not as versatile and flexible as our Python programs, it provides high accessiblity, guidance, and visualization of all evaluation processes. Topino and our Python programs generate comparable results. We can foresee more researchers and groups trying out and adopting Topino and our approach of MSS assessment using angulagrams due to removal of previous hurdles. Finally, we want to encourage these new users to contribute ideas or code to incrementally improve Topino and its assesment approach to advance the area of MSS evaluation even further.

\section{Availability and requirements}

Topino is publicly available 64bit software. It can be used,copied and distributed freely. Binaries for Windows and Linux (Debian package) can be downloaded from the Github repository. The Windows version runs on Windows 7 and 10, whereas the Linux version runs at least on Ubuntu $16+$ and Linux Mint 19+. Topino is released under the 3-Clause BSD and the source code is freely available on Github. This will allow other researchers and developers in the area of molecular-stream separations to contribute, implement, and extend analysis routines. Also, it is possible to compile and run the program on other platforms (e.g. Mac) than listed here.

Project name Topino

Project home page https://github.com/Schallaven/topino

Operating systems Windows 64bit $(7,10)$, Linux 64bit (tested on Ubuntu, Linux Mint)

Programming language $\mathrm{C}++17 / \mathrm{Qt5} /$ Eigen3

Other requirements No requirements

License 3-Clause BSD

Any restrictions to use by non-academics None

\section{Availability of data and materials}

Binary files, source code, and example files are available on the Github repository (https://github.com/Schallaven/topino, contributions are welcome!) Furthermore, snapshots are available as supplementary files on ChemRxiv.

\section{Competing interests}

The authors declare that they have no competing interests.

Funding

This work was supported by the Natural Sciences and Engineering Research Council (NSERC; STPG-P 521331-2018) of Canada.

\section{Author's contributions}

Sven Kochmann planned and developed the software. Nikita A. Ivanov helped with the development of the graphical user interface, bug fixing, and wrote the user guide. Sergey $N$. Krylov supervised the project and development and provided funding. All authors contributed to writing the paper. All authors read and approved the final manuscript.

\section{References}

1. Hilbrig, F., Freitag, R.: Continuous annular chromatography. Journal of Chromatography B 790(1), 1-15 (2003). doi:10.1016/S1570-0232(03)00085-0. Preparative Chromatography of Proteins

2. Novo, P., Janasek, D.: Current advances and challenges in microfluidic free-flow electrophoresis-a critical review. Analytica Chimica Acta 991, 9-29 (2017). doi:10.1016/j.aca.2017.08.017

3. Johnson, A.C., Bowser, M.T.: Micro free flow electrophoresis. Lab Chip 18, 27-40 (2018). doi:10.1039/c7lc01105a

4. Agostino, F.J., Cherney, L.T., Galievsky, V., Krylov, S.N.: Steady-state continuous-flow purification by electrophoresis. Angewandte Chemie International Edition 52(28), 7256-7260 (2013). doi:10.1002/anie. 201300104. https://onlinelibrary.wiley.com/doi/pdf/10.1002/anie.201300104
5. Jezierski, S., Tehsmer, V., Nagl, S., Belder, D.: Integrating continuous microflow reactions with subsequent micropreparative separations on a single microfluidic chip. Chem. Commun. 49, 11644-11646 (2013). doi:10.1039/C3CC46548A

6. Castro, E.R., Manz, A.: Present state of microchip electrophoresis: State of the art and routine applications. Journal of Chromatography A 1382, 66-85 (2015). doi:10.1016/j.chroma.2014.11.034. Editors' Choice IX

7. Pfeiffer, S.A., Rudisch, B.M., Glaeser, P., Spanka, M., Nitschke, F., Robitzki, A.A., Schneider, C., Nagl, S., Belder, D.: Continuous purification of reaction products by micro free-flow electrophoresis enabled by large area deep-uv fluorescence imaging. Analytical and Bioanalytical Chemistry 410(3), 853-862 (2018). doi:10.1007/s00216-017-0697-8

8. Islinger, M., Wildgruber, R., Völkl, A.: Preparative free-flow electrophoresis, a versatile technology complementing gradient centrifugation in the isolation of highly purified cell organelles. Electrophoresis 39(18), 2288-2299 (2018). doi: $10.1002 /$ elps. 201800187 https://onlinelibrary.wiley.com/doi/pdf/10.1002/elps.201800187

9. Preuss, J.-A., Nguyen, G.N., Berk, V., Bahnemann, J.: Miniaturized free-flow electrophoresis: production, optimization, and application using 3d printing technology. Electrophoresis n/a(n/a) (2020). doi: $10.1002 /$ elps. 202000149 https://onlinelibrary.wiley.com/doi/pdf/10.1002/elps.202000149

10. Jender, M., Novo, P., Maehler, D., Münchberg, U., Janasek, D., Freier, E.: Multiplexed online monitoring of microfluidic free-flow electrophoresis via mass spectrometry. Anal. Chem. 92(9), 6764-6769 (2020). doi:10.1021/acs.analchem.0c00996

11. Zhou, W., Xia, L., Xiao, X., Li, G., Pu, Q.: A microchip device to enhance free flow electrophoresis using controllable pinched sample injections. Electrophoresis 40(16-17), 2165-2171 (2019). doi:10.1002/elps. 201900079 https://onlinelibrary.wiley.com/doi/pdf/10.1002/elps.201900079

12. Staubach, S., Reiter, C., Weber, G., Giebel, B.: Free flow electrophoresis allows preparation of extracellular vesicles with high purity. Cytotherapy 21(5, Supplement), 57-58 (2019). doi:10.1016/j.jcyt.2019.03.432

13. Rudisch, B., Pfeiffer, S.A., Geissler, D., Speckmeier, E., Robitzki, A.A., Zeitler, K., Belder, D.: Nonaqueous micro free-flow electrophoresis for continuous separation of reaction mixtures in organic media. Anal. Chem. 91(10), 6689-6694 (2019). doi:10.1021/acs.analchem.9b00714

14. Zitzmann, F.D., Jahnke, H.-G., Pfeiffer, S.A., Frank, R., Nitschke, F. Mauritz, L., Abel, B., Belder, D., Robitzki, A.A.: Microfluidic free-flow electrophoresis based solvent exchanger for continuously operating lab-on-chip applications. Anal. Chem. 89(24), 13550-13558 (2017). doi:10.1021/acs.analchem.7b03959

15. Courtney, M., Thompson, E., Glawdel, T., Ren, C.L.: Counterflow gradient focusing in free-flow electrophoresis for protein fractionation. Anal. Chem. 92(10), 7317-7324 (2020). doi:10.1021/acs.analchem.0c01024

16. Herzog, C., Poehler, E., Peretzki, A.J., Borisov, S.M., Aigner, D., Mayr, T., Nagl, S.: Continuous on-chip fluorescence labelling, free-flow isoelectric focusing and marker-free isoelectric point determination of proteins and peptides. Lab Chip 16, 1565-1572 (2016). doi:10.1039/C6LC00055J

17. Kristoff, C.J., Bwanali, L., Veltri, L.M., Gautam, G.P., Rutto, P.K., Newton, E.O., Holland, L.A.: Challenging bioanalyses with capillary electrophoresis. Anal. Chem. 92(1), 49-66 (2020). doi:10.1021/acs.analchem.9b04718

18. Kochmann, S., Krylov, S.N.: Image processing and analysis system for development and use of free flow electrophoresis chips. Lab Chip 17(2), 256-266 (2017). doi:10.1039/C6LC01381C

19. Kochmann, S., Krylov, S.N.: Quantitative characterization of molecular-stream separation. Anal. Chem. 90(15), 9504-9509 (2018). doi:10.1021/acs.analchem.8b02186

20. Kochmann, S.: Schallaven/ffeimg: Image Processing and Analysis System for Development and Use of Free Flow Electrophoresis Chips. doi:10.5281/zenodo. 1001785

21. Kochmann, S.: Schallaven/angulagram: Quantitative Characterization of Molecular-stream Separation. doi:10.5281/zenodo.2592588 
22. Ivanov, N.A., Liu, Y., Kochmann, S., Krylov, S.N.: Non-aqueous continuous-flow electrophoresis (nacfe): potential separation complement for continuous-flow organic synthesis. Lab Chip 19(13), 2156-2160 (2019). doi:10.1039/C9LC00460B

23. Ivanov, N.A., Kochmann, S., Krylov, S.N.: Visualization of streams of small organic molecules in continuous-flow electrophoresis. Anal. Chem. 92(4), 2907-2910 (2020). doi:10.1021/acs.analchem.9b05734

24. Lu, N., Sticker, D., Kretschmann, A., Petersen, N.J., Kutter, J.P.: A thiol-ene microfluidic device enabling continuous enzymatic digestion and electrophoretic separation as front-end to mass spectrometric peptide analysis. Analytical and Bioanalytical Chemistry 412(15), 3559-3571 (2020). doi:10.1007/s00216-020-02609-5

25. The Qt Company: Qt : Cross-platform Application and UI Framework. https://www.qt.io/

26. Guennebaud, G., Jacob, B., et al.: Eigen v3 (2010). http://eigen.tuxfamily.org

27. Smith, K., Landes, P.-E., Thollot, J., Myszkowski, K.: Apparent greyscale: A simple and fast conversion to perceptually accurate images and video. Computer Graphics Forum 27(2), 193-200 (2008). doi:10.1111/j.1467-8659.2008.01116.x

Additional Files

Additional file 1 - Topino 1.0 Windows version

ZIP-file containing all files required to run Topino on Windows without installation.

Additional file 2 - Topino 1.0 Debian package

DEB-file for installation of Topino on Debian-based distributions (Ubuntu, Linux Mint, etc.)

Additional file 3 - Example images

ZIP-file containing a set of example images from our ChemRxiv preprints. 